\title{
Zur Lehre von der absoluten Muskelkraft.
}

\author{
Von \\ Cand. med. F. A. Feuerstein aus Aulendorf (Württemberg).
}

Unter absoluter Kraft eines Muskels verstehen wir nach Ed. Weber ${ }^{1}$ ) „dasjenige Gewicht, das er durch seine Contraction gar nicht zu heben vermag, welches aber auch umgekehrt ihn nicht zu dehnen vermag“. Denn würde der Muskel durch ein Gewicht gedehnt, so ist seine Kraft kleiner als das betreffende Gewicht; wïrde er andererseits das Gewicht heben, so ist seine Kraft grösser als die des Gewichtes. Denken wir uns das Gewicht unterstuitzt, so würden wir also dasselbe so lange zu vergrössern haben, bis der Muskel es gerade nicht mehr oder eben noch eine Spur von der Unterlage abhebt. Dass diese Grösse abhängig ist vom Querschnitt des Muskels oder, anders ausgedrückt, dass es abhängt von der Summe der einzelnen Fasern, liegt auf der Hand. Um daher verschiedene Muskeln mit einander vergleichen zu können, muss man wissen, welche Kraft ein als Einheit angenommener Querschnitt eines Muskels $(1 \mathrm{qcm})$ aufweist. Die gewöhnliche Art und Weise diesen Querschnitt zu bestimmen besteht darin, dass man das Volumen des Muskels durch seine Länge dividirt. Ersteres wird aus dem absoluten Gewicht des Muskels gefunden, indem man es durch sein specifisches Gewicht (1058) dividirt.

Wie man aber ohne Weiteres sieht, ist diese Bestimmung sehr ungenau; denn man macht die äusserst selten zutreffende Annahme, dass besagter Muskel aus lauter gleich langen parallelen Fasern besteht, eine Annahme, die vielleicht nahezu z. B. beim Sartorius des Frosches zutrifft. Von grösster Wichtigkeit für die Beurtheilung der Kraft eines Muskels ist aber die Summe seiner Fasern, die z. B. bei einem gefiederten Muskel ausserordentlich gross sein kann im Verhältniss zu einem dem Volumen nach gleichen Muskel,

1) Wagner's Handwörterbuch Bd. III, Thl. II, S. 84.

E. Pflüger, Archiv f. Physiologie. Bd. XLIII. 
der aber nur parallele Fasern hat, die alle so lang sind wie er selber. Wïrde man nun bei einem gefiederten Muskel den Querschnitt nach obiger von Weber angegebenen Regel berechnen, so erhielte man selbstverständlich einen viel zu geringen physiologischen Querschnitt; denn die Fasern sind eben nicht so lang wie der ganze Muskel und ihre Zahl ist ausserordentlich viel grösser als diejenige wäre, wenn die Fasern alle von der Länge des Muskels und einander parallel wären. Auch Henke ${ }^{1}$ ) hat Koster gegeniiber mit Recht auf den Unterschied des naturlichen Muskelquerschnitts dem physiologischen gegentiber hingewiesen und gezeigt, dass z. B. der physiologische Querschnitt des Gastroenemius eines erwachsenen kräftigen Mannes grösser sein kann als die Dicke des ganzen Unterschenkels, eine Thatsache, die Koster unmöglich schien.

Um ein wirklich brauchbares Urtheil tuber die absolute Kraft einer Muskelfaser ${ }^{2}$ ) zu gewinnen, müssten wir eine ganz genaue Kenntniss des Baues eines Muskels haben und zum Mindesten die mittlere Länge und Anzahl der Fasern kennen. Die Schwierigkeiten dieser Kenntniss werden aber noch dadurch bedeutend vermehrt, dass die verschiedenen Fasern eines Muskels unter sich weder vollkommen gleich, noch auch parallel sind und dass in sehr vielen Muskeln bindegewebige Massen (Inscriptionen, Aponeurosen u. s. w.) nach mannigfachen Richtungen eingelagert sind.

Wenn man sich also über die verschieden grosse Kraft zweier gleich langer und gleich schwerer Muskeln ein Urtheil verschaffen will, so würde man, selbst wenn diese Kraft sehr verschicden ausfiele, über die Kraft der sie zusammensetzenden einzelnen Muskelfasern nichts erfahren. Nur dann könnte man behaupten, dass die einzelnen einen Muskel zusammensetzenden Fasern sehr verschieden in ihrer Kraft sind, wenn die Muskeln auch gleichartig gebaut sind.

Eine weitere Einsicht in die Grösse der Kraft von zwei gleich grossen und gleich langen Muskeln, insoweit dieselbe auf die ein-

1) Zeitschrift für rationelle Medicin, dritte Reihe Bd. 33, S. 148.

2) Mit Bezug auf die neuestens von Fick (dieses Archiv Bd. 41, S. 176) constatirte grössere Reizwirkung der willkürlichen Innervation gegenüber der faradischen Erregung ist hier zu erwähnen, dass sich meine Arbeit wesentlich auf elektrische Reizung des Muskels bezicht und derartige Vergleichsbestimmungen nicht enthält. 
zelne Faser zu beziehen ist, kann man sich auch dadurch verschaffen, dass man die Verkürzung misst, die der Muskel bei einer maximalen Reizung aufweist. Ist dieselbe bedeutend, so würde der Muskel aus verhältnissmässig langen, ist sie unbedeutend, so würde der Muskel aus verbältnissmässig vielen und kurzen Fasern bestehen. Hierbei wäre natürlich anzunehmen, dass sich eine lange und eine kurze Muskelfaser bei maximalem Reiz um gleich viel ihrer ursprünglichen Länge, also jede etwa auf die Hälfte verkürzte und weiterhin auf die Grösse des Winkels Rücksicht zu nehmen, den die Richtung der Fasermasse des Muskels mit der Längsrichtung derselben bildet. Je grösser dieser Winkel, von $0^{0}$ bis etwa $45^{\circ}$ wachsend, ist, um so unbedeutender würde natürlich bei gleicher Faserlänge die Verkürzung des Muskels ausfallen.

Betreffs des historischen Theils unserer Frage verweise ich auf Hermann's Handbuch der Physiologie der Bewegungsapparate I. Bd. S. 61 .

Nur kurz gedenken möchte ich des Weber'schen Versuchs and der an ihn sich unmittelbar anschliessenden Betrachtungen. Ed. Weber fand pro $1 \mathrm{qcm}$ menschlicher Wadenmuskeln die absolute Kraft im Durchschnitt zu $1 \mathrm{~kg}$. Der Hebelarm, an welchem nach dem genannten Autor die Kraft der Wadenmuskeln wirkt, reicht vom Drehpunkt des Zehengelenkes bis zum Ansatzpunkt der Achillessehne an der Ferse; der Hebelarm, an welchem die Last des Körpers wirkt, dagegen von dem Drehpunkt des Zehengelenkes nur bis zur Axe des Fussgelenkes im Sprungbein. Henke und Knorz ${ }^{1}$ ) corrigirten die Weber'schen Annahmen, welche nur dann Sinn hätten, wenn der Zug der Muskeln von einem ausserhalb des Körpers liegenden festen Punkte ausgienge, was ja aber doch in Wirklichkeit nicht stattfindet. Diese beiden Forscher zeigten, dass der Hebelarm der Kraft ein viel kleinerer ist, nämlich der kürzeste Abstand der Achillessehne von der Axe der Talusrolle. Dem 4mal kleineren Hebelarm der Kraft entsprechend fanden sie die absolute Kraft pro 1 qcm menschlicher Wadenmuskeln auf Grund der Weber'schen Zahlen 4mal grösser, im Mittel also zu $4 \mathrm{~kg}$. Auf Grund eigener Versuche bestimmten sie weiter für die Dorsalflexoren des Fusses die absolute Kraft zu ca. $6 \mathrm{~kg}$.

1) Zeitschrift f. rationelle Medicin; dritte Reihe, Bd. 24, S. 247. 
pro 1 qcm, für die Flexoren des Vorderarms zu je $8-9 \mathrm{~kg}$. Hermann hat in seinem Handbuch der Physiologie der Bewegungsapparate Bd. I, S. 64 die Resultate der verschiedenen Forscher zusammengestellt wie folgt:

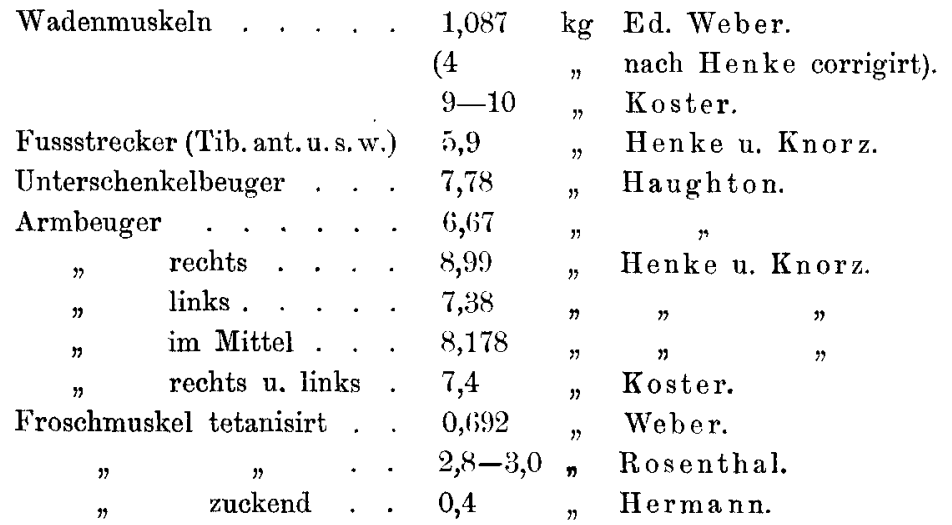

Um nun einen genaueren Einblick in die Vorgänge zu gewinnen, welche sich vollziehen, wenn wir uns durch Zusammenziehen der Wadenmuskeln auf die Zehen erheben, um namentlich die Art und Grösse der Verkịrzung der Muskeln, sowie die Art und Grösse des Hubes unseres Körpers, also ihre Leistung ${ }^{1}$ ), genau festzustellen, wurde ein Modell des menschlichen Untersehenkels angefertigt, an welchem man die jedesmalige mittlere Länge des Wadenmuskels, der durch eine Stablfeder dargestellt war, leicht messen konnte. Dieselbe verkleinerte sich natiurlich, wenn das Ende der Tibia sich hob und in dem Fussgelenk des Modells eine Drehung stattfand.

Das Modell war, wie nebenstehende Skizze (s. Fig. 1) zeigt,

1) Hierüber existiren z. Th. merkwürdige Angaben in der Litteratur. Obwohl der Satz Galens (Musculi cum insigni virium detrimento agunt) durchaus richtig ist, zeigt doch folgende aus der Anatomie von Hyrtl (8. Aufl. 1863, S. 101) entnommene Angabe, dass man sich hierüber doch auch irrige Vorstellungen gemacht hat. Die Stelle lautet: „Um ein erklärendes Beispiel zu geben, führe ich an, dass die Wadenmuskeln eines Menschen, der auf einem Fuss stehend, sich auf die Zehenspitze erhebt, 80 mal mehr Kraft entwickeln müssen, als ihre Wirkung eigentlich beträgt, dass sie also statt 140 Pfunden, die wir als mittleres Gewicht eines erwachsenen Mannes annehmen, in Wahrheit ein Gewicht von 11200 Pfunden tragen." Ich weiss nicht, auf welche Versuche Hyrtl diese Angaben stützt. Weiter unten aber wird gezeigt werden, wie weit da über das Ziel hinausgeschossen worden ist. 
folgendermassen gebaut. Auf einem festen Dreifuss war eine in Stahlspitzen laufende horizontale Axe $A$ befestigt, um welche sich das Winkelstiick $A B C$. drehte. $A$ stellt die Spitze des ersten Metatarsuskopfes dar, $B$ die Mitte des Fussgelenks, $C$ die Ferse. Ein auf $B C$ verschiebbares Häkchen diente zur Befestigung der den Wadenmuskel darstellenden Feder. In $B$ war die Tibia $B D$ in einer ähn. lichen Axe, wie $A B C$ in $A$ eingelenkt. Auch sie trug an ihrem oberen Ende ein verschiebbares Häkchen und ebenda pa-

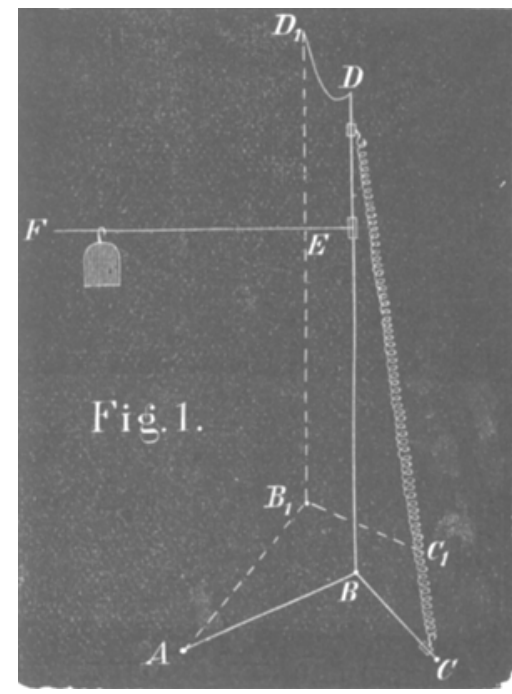
rallel der Axe des Fussgelenks mehrere Löcher, die zum Visiren bestimmt waren. An ihrem unteren Ende $B$ hat die Tibia vorn und hinten mit Schrauben einstellbare Anschläge, so dass man sie leicht nicht bloss nach vorn und hinten um sehr kleine Winkel drehen, sondern auch befestigen kann. Die Stangen des Modells $A B C$ und $B D$ waren hohle Messingröhren und maassen $A B, B C$, $B D$ bezüglich $13,5,9,0,37,0 \mathrm{~cm} \Varangle A B C=121^{\circ} 30^{\prime}$.

Das Modell giebt eine klare und bubsche Vorstellung von der Art und Weise, wie die Wadenmuskeln beim Hube des Körpers wirken. Spannt man nämlich eine gedehnte Feder zwischen $C$ und $D$ aus, so wird, falls man ein Unkippen des Modells durch ein leichtes Auflegen des Fingers auf $D$ oder auf andere Weise verhindert, der Finger nach oben gedrückt, indem $D$ in die Höhe steigt und $\Varangle C B D$ sich verkleinert. Das Modell nimmt hierbei die Stellung $A B, C, D$, ein. Für derartige Versuche ist es, nebenbei bemerkt, zweckmässig, die Schrauben der Axen in $A$ und $B$ ein wenig anzuziehen, vorausgesetzt, dass bei entsprechender Belastung des Modells, die man am besten an einer auf $B D$ nach vorn angebrachten Stange $E F$ als Laufgewicht befestigt, das Modell ähnlich wie ein auf den Zehen stehender Mensch im labilen Gleichgewioht feststehen soll. Hierbei muss natürlich der Schwerpunkt des ganzen Systems auf dem in $A$ über $A C$ errichteten Perpendikel liegen. 
Das Modell sollte also eine einigermaassen genane Vorstellung geben von der jeweiligen Länge der Wadenmuskeln bei verschiedener Stellung der Tibia in dem Fussgelenk. In zweiter Linie aber kam es nun wesentlich darauf an zu wissen, welche Bewegung eben die Tibia in dem Fussgelenk macht, wenn wir uns auf die Zehen heben. Hierzu war nöthig, die Bewegung eines Punktes der Tibia, am besten eines dem Knie nahe gelegenen zu bestim. men, wenn man sich auf die Zehen hebt, Kennt man dessen Weg, so ergiebt sich unmittelbar die hierbei stattfindende Veränderungr des Wadenmuskels, vorausgesetzt, dass es eben angeht, die Verhältnisse des Modells auf die des menschlichen Körpers zu übertragen, was aber jedenfalls ohne nennenswerthe Fehler geschehen darf. Man hat ja nur das Knieende der Tibia im Modell ganz denselben Weg zuriicklegen zu lassen, den dieses Ende desselben Knochens an der Versuchsperson zurückgelegt hat, wenn sie sich auf die Zehen hebt.

Zu diesem Zweck verfuhr ich auf Anrathen von Herrn Prof. Grützner folgendermaassen: Die Versuchsperson stellte sich mit entblössten Schenkeln in einem verdunkelten Zimmer möglichst nahe an eine weisse vertical stehende Papierwand auf. In einer Entfernung von etwa $5 \mathrm{~m}$ stand in gleicher Hïhe wie das Knie der Versuchsperson und genau seitlich von ihr, also weder vor noch hinter ihr, eine hell brennende Lampe, welche ein scharfes und genaues Profil der Beine der Ver-

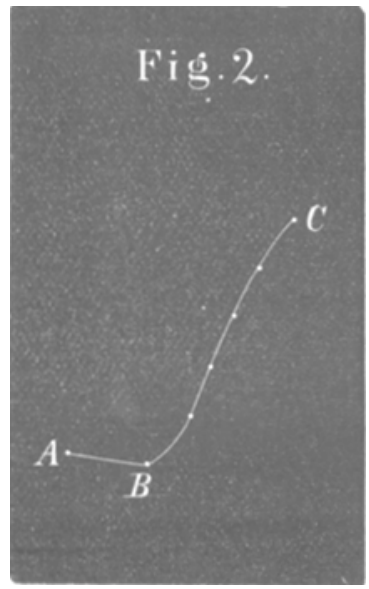
suchsperson an die weisse Papierwand warf. Nun wurde ein Punkt der Crista tibiae dicht nnter dem Knie durch ein darauf geklebtes, mit einer Spitze versehenes Stäbchen markirt. Jetzt erhob sich die Versuchsperson bis zu dem Punkt, wo eine weitere Erhebung nicht mehr möglich war. Waihrend dieses Vorgangs hatte ein Assistent die Bewegungen, welehe der Schatten der Spitze machte, sofort auf dem Papier nachzuzeichnen. Auf diese Weise erbielt ich etwa folgende, auf die Hälfte verkleinerte Curve $A B C$ (s. Fig. 2).

Das Knieende der Tibia bewegt sich also zuerst nach vorn 
und ein wenig nach unten von $A$ nach $B$. Während dieser Zeit beugen wir nänilich den in den Knie- und Hüftgelenken abgesteiften Körper ein wenig nach vorn durch Drehung in dem Fussgelenk und verlegen hierdurch den Schwerpunkt über die Metatarsusköpfe. Während dieser ganzen Zeit $(A B)$ hat sich die Ferse noch nicht vom Boden erhoben. Die Hebung der Ferse und damit des ganzen Körpers beginnt erst von $B$ ab und geht dann mit nahezu gleichmässiger Geschwindigkeit weiter von statten von $B$ nach $C$. Der ganze Hub beträgt hiernach im Mittel etwa $5 \mathrm{~cm}$. Man wïrde vielleicht meinen, dies sei zu gering. Allein wir haben uns vielfach davon überzeugt, dass mittelgrosse Personen sich nur um diese Grösse emporheben, wenn sie sich auf die Zehen stellen.

Nun kam es darauf an, das obere Ende der Tibiastange des Modells, welches, wie schon erwähnt, mit einem Visir versehen war, die gleiche Curve beschreiben zu lassen, welche der Schatten der Stäbchenspitze ergeben hatte. $\mathrm{Zu}$ dem Zweck wurde das Modell der auf einer verticalen Wand gezeichneten Curve in paralleler Richtung nahe gegenübergestellt und nun das Visir nacheinander auf diejenigen Punkte der Curve, deren verticaler Abstand immer $1 \mathrm{~cm}$ betrug, eingestellt und bei jeder Einstellung die Entfernung des obern Endes der Tibiastange von dem Fersenhöcker (die Gastrocnemiuslänge) gemessen. Hierbei zeigte sich, dass sich die Wadenmuskeln, um den Körper den ersten Centimeter in die Höhe zu heben, etwa um $5 \mathrm{~mm}$ verkürzen mussten. Für den nächsten Centimeter Hub verkurzten sie sich um 6 , weiter um 6,8 und schiesslich, um den Körper um den letzten der $5 \mathrm{~cm}$ zu erheben, um $9 \mathrm{~mm}$. Nach bekannten Gesetzen der Mechanik (Gesetz von den virtuellen Geschwindigkeiten) verhält sich hiernach die Kraft des Muskels in ihrem Beginn (erster Hub) zur Last des Körpers wie $10: 5$, in ihrem Ende (letzter, fünfter Hub) wie 10:9.

Der Wadenmuskel (beziehungsweise der linke und der rechte zusammen) entwickelt also im Anfang seiner Verkürzung die grösste Spannung. Dieselbe ist - auch wenn diese Zahlen keineswegs auf absolute Genauigkeit Anspruch erheben können noch sollen - während des Hubes um den ersten Centimeter, gleich der doppelten Körperlast, am Ende seiner Zusammenziehung, die im Ganzen also etwa $3,4 \mathrm{~cm}$ betragen wird, dagegen nahezu nur gleich der einfachen. Es entwickelt also der Wadenmuskel im Beginn seiner Verktirzung die grösste, am Ende derselben die geringste Spannung. Diese be- 
kanntlich schon von Schwann für isolirte Muskeln experimentell festgestellte Thatsache lässt sich auch unmittelbar, wic es Fick ${ }^{1}$ ) gethan hat, an dem von uns genauer beschriebenen Vorgang des Erhebens auf die Zehen zeigen, indem sich während des Erhebeus der Ferse der Hebel der Kraft etwas vergrössert, der der Last aber nahezu constant bleibt. Eine genaue Feststellung dieser Verhältnisse und verschiedener Hebellängen an einer Versuchsperson ist aber natiirlich ohne Weiteres nicht möglich.

Aus obiger Curve lässt sich noch ein weiteres für alle Muskeln giiltiges Gesetz demonstriren. Gehen wir von Punkt $A$ Fig. 2 aus, so sieht man, wie schon oben erwähnt, dass sich das Knicende der Tibia ein wenig voriiber beugt nach $B$ Fig. 2. Hierdurch wird, wie ohne Weiteres einzusehen (siehe Fig. 1), der Punkt $C$ von $D$ durch Vergrösserung des Winkels $C B D$ entfernt, der Wadenmuskel also passiv gedehnt und stärker angespannt. Erst nach dieser Dehnung erfolgt die Innervation des Muskels und die damit Hand in Hand gehende Zusammenziehung.

Gedenken wir noch kurz der an Froschmuskeln angestellten Versuche über die Muskelkraft, so wurde hierzu das von Helml oltz eingeführte Verfabren der Ueberlastnng angewandt. Namentlich Rosenthal ${ }^{2}$ ) bediente sich desselben. Dils an den Muskel befestigte Gewicht wurde anf irgend eine Weise zunächst gretragen und erst wenn der Muskel sich zusammenzog, uibte es einen Zug auf denselben aus. Dasjenige Gewicht nun, welches der gereizte Muskel hierbei nicht mehr oder eben noch ein wenig erheben konnte, war seine absolute Kraft.

Anschliessend an das Helmholtz'sche Ueberlastungsverfahren fuibrte ich auf Anregung und unter Anlcitung von Herrn Professor Grïtzner mit dieser wie man glauben sollte, fehlerfreien Methode eine Reihe von Kraftbestimmungen an verschiedenen Muskeln des Frosehes und der Kröte aus. Nachdem wir im Allgemeinen die früheren Angaben von Rosenthal, Hermann, Bernstein u. a. bestätigen konnten, waren wir erstaunt zu sehen, dass bei nur geringfiigig veränderten Versuchsbedingungen die bei einer Zuckung erzeugte absolnte Muskelkraft sich häufig ausserordentlich viel grösser erwies, als unsere und anderweitige Beobachtungen ergaben.

1) II ermann, Bd. I, zweite Abthl., S. 282.

2) Comptes rendus, T. 64 , S. $1143,1867$. 
Man konnte die Ursache dieser ausserordentlich verschiedenen Erfolge sehr bald in einer mangelhaften Versuchstechnik finden; vor allen Dingen zeigte sich, dass die bei einer Zuckung a u rtretende absolute Kraft unmittelbar abhängig war von der Spannung, die dem Muskel unmittelbar vor seiner Reizung ertheilt wurde. Wie nachträglich genauer mitzuthcilende Versuchsergebnisse lehren werden, schwankten die Grössen der absoluten Kraft zwischen dem Ein- bis Zehnfachen und die Ursache dieser verschiedenen Versuchsergebnisse lag eben darin, dass die Anfangsspannung dès Muskels, von welcher man ausging und die gleich Null sein sollte, eben nicht gleich Null war, sondern stets eine gewisse, aber wechselnde positive Grösse hatte.

Da in dieser verschiedenen Kraftleistung der Muskeln offenbar eine physiologisch wichtige und ungemein zweckmässige Einrichtung gegeben ist, habe ich diese Angelegenheit im Tübinger physiologischen Institute des Genaueren weiter verfolgt und lege die Ergebnisse dieser meiner Untersuchungen in Folgendem kurz dar.

\section{Eigene Versuche.}

\section{A. Methode.}

Während alle friiheren Beobachter, welche zur Bestimmung der Muskelkraft die Ueberlastungsmethode anwendeten, von der Anfangsspannung Null ausgingen oder auszugehen glaubten ${ }^{1}$, kam es uns jetzt darauf an, J) die Zuckung des Muskels von einer willkürlich zu verändernden Anfangsspannung aus vor sich gehen zu lassen und 2) die bei jener Zuckung stattfindende maximale Spannung zu messen.

Der von Herrn Prof. Grützner angegebene und von Mechanikus Himmel hierorts selbst tadellos angefertigte Apparat, welcher jenen beiden oben genannten Anforderungen entsprach, war etwa folgendermaassen gebaut (siehe Fig. 3, S. 356):

Auf einem festen und schweren Grundbrett von Blei $(A B)$ steht zur Rechten des Arbeiters ein Stab mit einer horizontalen

1) Thatsächlich wird nämlich dem Muskel selbst bei Ueberlastungsversuchen immer eine bestimmte und häufig gar nicht zu unterschätzende Anfangsspannung crtheilt, wie uns vielfache Versuche an dem weiter unten zu beschreibenden Apparate gelehrt haben. 
Muskelklemme $(K)$, welche den zu untersuchenden Muskel an einem passend hergerichteten Knochenstïck festhält und leicht nach links

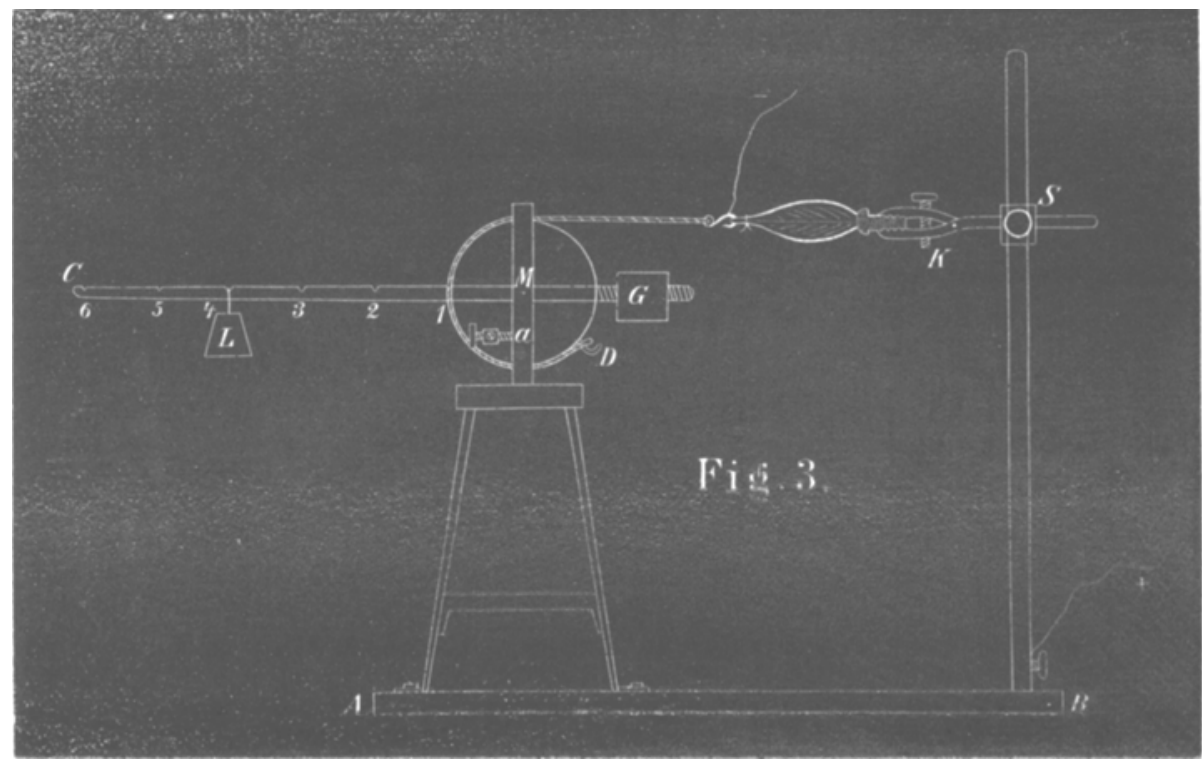

und rechts verschoben und durch die Schraube $s$ fixirt werden kann. Dem Muskelhalter gegenüber befindet sich eine leichte feste Rolle von 4-5 cm Durchmesser, welche sich um eine horizontale senkrecht zum Muskelzug stehende Axe (MI) dreht. Der horizontale Durchmesser dieser Rolle trägt an dem den Muskel zugewendeten Ende bezw. seiner Verlängerung ein äquilibrirendes Gewicht $(G)$; nach der andern Seite ist der Durehmesser verlängert in einen Stab $(M C)$, der wie der Wagbalken einer Schnellwage in gleiche Theile abgetheilt und mit entsprechenden Zahlen (von 1--6) versehen ist. Zudem hat er in seiner oberen Fläche kleine Einschnitte, welche ein an ihm hin und her zu schicbendes Laufgewicht $(L)$ festhalten.

Um den grössten Theil der Rolle herum ${ }^{1}$ ) liegt in ihrer Rinne ein unausdehnbarer, in passender Weise bei $D$ befestigter Faden, der, mit entsprechender Kraft angezogen, die Rolle rechts

1) Dieser Apparat ist auch für die Versuche an Säugethiermuskoln bestimmt; deshalb ist der Faden horizontal nach dem Muskel gefïhrt, weil die 
herumdrebt und dabei das Gewicht $(L)$ in die Höhe bebt. Das Ende des Fadens ist, wie leicht ersichtlicb, mit der Sehne des Muskels, z. B. des Gastrocnemins, verknuipft, wobei, was hier gleich vorweg zu bemerken, jede auch noch so geringe Verletzung des Muskels zu vermeiden ist. Wollte man etwa wie bei andern Muskelversuchen einen Haken durcb den Gastrocnemius oberhalb der Achillessehne einlegen, so würde schon bei mässigen Anspannungen und Leistungen des Muskels der Muskel zerreissen.

Vermittelst des jetat besehriebenen Apparates, der zugleich noch so eingerichtet ist, dass der mit dem Gewicht beschwerte Hebelarm in Folge eines Anschlages an der Rolle (a) horizontal stehen bleibt, ist es natürlich leicht die absolute Kraft eines Muskels unter den versebiedensten Bedingungen zu bestimmen. Man hat den Muskel eben nur, wie oben beschrieben, in demselben zu befestigen, so dass er die Spannung Null oder nahezu Null besitzt und ihn mit elektrischen Strömen mittelbar oder unmittelbar zu reizen. Hierbei wird er, wenn das Laufgewicht zu klein ist oder zu nahe dem Mittelpunkt der Rolle hängt, dasselbe hoch in die Höhe heben und die Rolle stark drehen; sehieht man es aber weiter vom Mittelpunkt weg, so wird man sehr bald nach wenigen tastenden Versuchen eine Gewichtsgrösse finden, die der Muskel gerade noch ein wenig zu heben im Stande ist. Es gibt sich das regelmässig durch ein klappendes Geräusch zu bemerken, welches entsteht, wenn die auch nur ein ganz klein wenig von dem Anschlag (a) abgehobene und beschwerte Rolle bei Erschlaffung des Muskels wieder anf den Anschlag zurïckfällt.

Jetzt handelt es sich noch weiter darum, die Anfangsspannung des Muskels nicht bloss Null sein zu lassen, sondern sie beliebig verändern und messen zu können. Das gescbieht folgendermaassen: Wenn der Muskel mit dem Faden an der Rolle verknüpft ist, hängt man bei horizontaler Lage von $G C$ ein bestimmtes Gewicht an den Hebelarm und entfernt jetzt die Muskelklemme so weit

Muskeln der Säugethiere wohl nur in dieser Lage sich bequem untersuchen lassen. Für die Versuche an Froschmuskeln dürfte es sich vielleicht empfehlen, die Schnur von Punkt 1 senkrecht emporsteigen zu lassen und den Muskel senkrecht darüber zu befestigen, was durch ein Heranrücken des die Muskelklemme tragenden Stabes nach links an die Rolle sich leicht bewerkstelligen lässt. 
nach rechts, bis sich der Contact in a eben abhebt. Nun ist der Muskel mit dem entsprechenden Gewicht bezw. je nachdem es in 2, 3 oder 6 hängt mit dem 2-, 3- bis 6fachen des angehängten Gewichts angespannt und behält fortwährend diese Spaunung, anch wenn an den Hebelarm jetzt andere und viel grössere Gewichte angebängt werden, weil der Anschlag eine stärkere Spannung des Fadens verhindert. Hierbei ist es erstaunlich zu sehen, dass selbst mittlere Gewichte bis zu $5 \mathrm{gr}$ den an dem Muskel befindlichen Faden noch ausserordentlich schlaff lassen, so dass man glauben möchte, er sei gar nicht gespannt. Wie man also sieht, vereinigt der Apparat in sich die Methoden einer in weiten Grenzen leicht zu verändernden Belastung und Ueberlastung.

Der Versuch gestaltet sich hiernach folgendermaassen: Der in der Muskelklemme an einem Knochen festgehaltene Muskel wird in oben beschriebener Weise mit dem Faden der Rolle in Ver. bindung gebracht, wobei, wie nochnals hervorzulseben, jede auch noch so geringfügige Verletzung des Muskels zu vermeiden ist. Vielfach wird man daher den Muskel auch nicht mit seiner Sehne an den Haken befestigen, sondern, wic z. B. beim Sartorius des Frosches auch entsprechende Knochenstïcke, an denen sich die Sehne ansetzt, daran lassen.

In der Muskelklemme ist, wie sich ziemlich von selbst versteht, der eine Zuleitungsdraht (siehe + Fig. 3) befestigt, wälrend der andere - mit dem Haken an dem Faden der Rolle verbunden ist. Der letztere Draht ist, um nicht den Muskel zu beschweren, möglichst leicht und dünn zu nehmen.

Nun beginnt man mit einer möglichst geringen Spannung des Muskels und reizt durch einen mässig starken Induktionsschlag (gewöhnlicher Induktionsapparat mit Eisenkern, ein Daniell, Rollenabstand $10 \mathrm{~cm}$ ). Nach kurzem Herumprobiren wird man die Grösse des Gewichts feststellen können, das gerade noch soweit gehoben wird, dass der Hebel sich von dem Anschlag, wenn auch nur eine Spur, entfernt. Vergrössert man die Anfangsspannung des Muskels und lässt etwa jetzt dasselbe Gewicht an derselben Stelle, so wird man sehen, wie bei wiederholter gleichartiger Reizung des Muskels das nämliche Gewicht mit Leichtigkeit ziemlich hoch gehoben wird. Man kann das Gewicht bedeutend vergrössern, um eine geringfijgige, eben bemerkbare Hebung desselben zu beobachten, mit Einem Wort: Die absolute Kraft des Muskels ist bedentend 
gewachsen. So geht es eine Weile fort, bis man an die endgültige Grenze angekommen ist, $\mathrm{d}$. $\mathrm{h}$. bis man die maximale absolute Kraft bei einer einmaligen Zuckung festgestellt hat.

Wird jetzt der Muskel tetanisirt, so ist die nun zu beobachtende absolute Kraft in der Regel noch etwas grösser, als die maximale absolute Kraft bei der Zuckung. Uebrigens verwischen sich die Leistungsunterschiede in Zuckung und Tetanus um so mehr, je mehr sich der Muskel dem Maximum der Spannung nähert.

$\mathrm{Zur}$ genaueren Illustrirung jener oben ausgesprochenen Bebauptung erlaube ich mir aus meinem Versuchsprotokoll eine Reibe von Versuchen folgen zu lassen mit methodisch gesteigerten Spannungsverbältnissen des Muskels.

\section{B. Versuche.}

1. Versuch.

4. Mai 1887. Gastrocnemius der Kröte; I Länge des Muskels $11 \mathrm{~mm}$, Gewicht $0,18 \mathrm{gr}$; Rollenabstand $5 \mathrm{~cm}$.

Bei Spannung des Muskels mit $10 \mathrm{gr}$ betrug das abgehob. Gew. $45 \mathrm{gr}$,

Im Tetanus

$\begin{array}{rll}20 " & " & 100 " \\ 40 " & " & 136 " \\ 80 " & 150 " \\ 100 " & 162 " \\ 200 " & 200 " \\ & " & 325 "\end{array}$

2. Versuch.

Gastrocnemius Rana esculenta; Länge des Muskels $30 \mathrm{~mm}$, Gewicht 0,8 gr.

Bei Spannung des Muskels mit $10 \mathrm{gr}$ betrug das abgehob. Gew. $130 \mathrm{gr}$,

Im Tetanus "

$\begin{array}{rlr}20 " & " & 220 " \\ 40 " & " & 300 " \\ 80 " & 350 " \\ 150 " & 380 " \\ 200 " & 425 " \\ 800 " & 800 " \\ & & 900 " \\ & & \text { (der Muskel reisst ein). }\end{array}$

3. Versuch.

3. Mai 1887. Grosse weibliche Rana esculenta; Gastrocnemius mit Nerv; von diesem aus gereizt; Fadenschlinge oberhalb der Achillessehe; Länge des Muskels $17 \mathrm{~mm}$; Gewicht 1,25 gr; Induktionsrolle im Abstand ron $10 \mathrm{~cm}$. 
Bei Spannung des Muskels mit $10 \mathrm{gr}$ betrug das abgehob. Gew. $200 \mathrm{gr}$,

$20 "$
$40 "$
80
$100 "$
$500 "$
300 D 400 . 475 D 525 จ 650 ๖

Im Tetanus $700 \nu$

4. Versuch.

Rana esculenta. Semimembranosus.

Gowicht $0,57 \mathrm{gr}$; Länge $28 \mathrm{~mm}$; der Muskel am Knochon befestigt; Versuchsdauer 12 Minuten.

Bei Spannung des Muskels mit $10 \mathrm{gr}$ betrug das abgehob. Gew. $180 \mathrm{gr}$,

$20 "$
$40 "$
$80 "$
100
$200 "$
400
212 212 " 212 " 212 ఎ 270 " Im Tetanus ,

\section{Versuch.}

Rectus abdominis dessolben Frosches, doppelt, durch cincn Faden befestigt; Gewicht $0,45 \mathrm{gr}$; Länge $37 \mathrm{~mm}$; Versuchsdauer 20 Minuten; Rollenabstand $8 \mathrm{~cm}$.

Bei Spannung des Muskels mit 10, gr betrug das abgehob. Gew. $22 \mathrm{gr}$,

\begin{tabular}{|c|c|c|c|c|}
\hline 》 & 20 & " & " & 30 \\
\hline n & 30 & $»$ & " & 35 \\
\hline נ’ & 40 & $"$ & " & 45 \\
\hline 》 & 50 & $\nu$ & " & 55 \\
\hline x & 60 & , & " & 65 \\
\hline 》 & 70 & " & $D$ & 70 \\
\hline D & 80 & " & $n$ & 80 \\
\hline$"$ & 90 & $D$ & $v$ & 90 \\
\hline " & 100 & 》 & $"$ & 100 \\
\hline
\end{tabular}

\section{Versuch.}

5. Juli 1887. Rana esculenta, Sartorius; Länge $35 \mathrm{~mm}$; Gewicht $0,25 \mathrm{gr} ;$ Haken durch die Symphyse, unteres Ende in der Knochenzange; Rollenabstand $10 \mathrm{~cm}$.

Bei Spannung des Muskels mit $10 \mathrm{gr}$ betrug das abgehob. Gew. $35 \mathrm{gr}$,

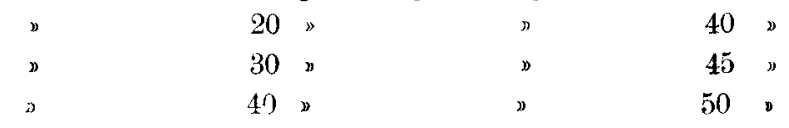


Bei Spannung des Muskels mit $50 \mathrm{gr}$ betrug das abgehob. Gew. $63 \mathrm{gr}$,

\begin{tabular}{|c|c|c|c|c|}
\hline & " & 60 & $D$ & 65 \\
\hline & , & 70 & $D$ & 75 \\
\hline & " & 80 & 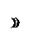 & 82 \\
\hline & $n$ & 90 & " & 90 \\
\hline Im Tetanus & y & & $n$ & 100 \\
\hline
\end{tabular}

7. Versueh.

7. Juli 1887. Mittelgrosse Rana esculenta. Muskeln weisslich trübe, zahlreiche punktförmige Ecchymosen. Sartorius wie früher präparirt, Haken in der Symphyse. Rollenabstand $10 \mathrm{~cm}$. Gewicht 0,24 gr. Länge $33 \mathrm{~cm}$.

Bei Spąnnung mit $10 \mathrm{gr}$ wurden gehoben $35 \mathrm{gr}$,

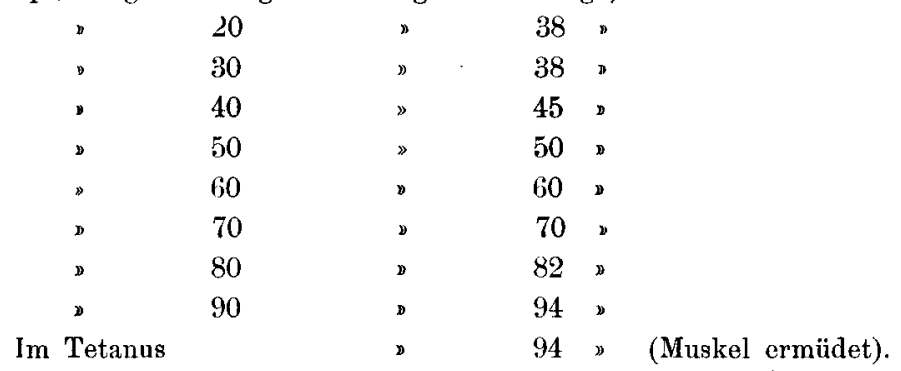

8. Versuch.

13. Juli 1887. Rana esculenta. Tibialis anticus; Gew. $0,15 \mathrm{gr}$; Länge $25 \mathrm{~mm}$; Rollenabstand $10 \mathrm{~cm}$.

Bei Spannung mit $10 \mathrm{gr}$ wurden gehoben $43 \mathrm{gr}$,

\begin{tabular}{|c|c|c|c|}
\hline & 15 & D & 67 \\
\hline , & 20 & & 75 \\
\hline & 30 & D & 80 \\
\hline
\end{tabular}

9. Versuch.

Kleine Rana esculenta (Weibchen); Muskeln weisslich trübe. Gastrocnemius; Länge $22 \mathrm{~mm}$; Gewicht $0,45 \mathrm{gr}$; Rollenanstand $5 \mathrm{~cm}$.

Bei Spannung mit $10 \mathrm{gr}$ wurden gehoben $200 \mathrm{gr}$,

\begin{tabular}{|c|c|c|c|}
\hline 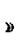 & 20 & , & 225 \\
\hline , & 40 & ๖ & 275 \\
\hline D & 80 & v & 300 \\
\hline 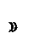 & 100 & D & 300 \\
\hline
\end{tabular}

10. Versuch.

Kröte. Gastrocnemius. Länge $18 \mathrm{~mm}$; Gewicht $0,3 \mathrm{gr}$; Rollenabstand $5 \mathrm{~cm}$. 
Bei Spannung mit $10 \mathrm{gr}$ wurden gehoben $85 \mathrm{gr}$,

$\begin{array}{lrlll}" & 20 & & 125 & \\ " & 40 & * & 150 & \\ " & 80 & & 185 & \\ " & 150 & & 230\end{array}$

Ein ähnliches Anwachsen der absoluten Kraft wurde festgestellt für den Biceps, Hyoglossus und Quadriceps des Frosches; letzterer ist übrigens für diese Versuche" weniger geeignet, da es sehr schwer ist, den frei präparirten Muskel ganz oder auch nur den grössten Theil seiner Fasern gleichmässig anzuspannen.

Versuche, ob gewisse Nervengifte, z. B. Curare oder Aether, an unserem Gesetz etwas ändern, haben negative Resultate crgeben.

\section{Versuch.}

Rana esculenta, weiblich. Diesem Frosch wurde die Art. iliaca einer Seito unterbunden und nun das Thier stark curarisirt. Auf diese Weise hat man an dem nicht curarisirten Gastrocnemius der unterbundenen Seite ein gutes Controlpräparat.

Curarisirter Gastrocnemius; Länge $21 \mathrm{~mm}$; Gewicht $0,23 \mathrm{gr}$.

Bei Spannung mit $10 \mathrm{gr}$ wurden geholen $110 \mathrm{gr}$,

\begin{tabular}{|c|c|c|c|}
\hline " & 20 & y & 120 \\
\hline " & 40 & " & $16 ; 0$ \\
\hline 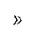 & 80 & $"$ & 220 \\
\hline " & 150 & » & 240 \\
\hline " & 200 & D & 250 \\
\hline " & 400 & $"$ & 440 \\
\hline & & & 450 \\
\hline
\end{tabular}

Anderer nicht curarisirter Gastrocnemius desselben Frosches.

Bei Spannung mit $10 \mathrm{gr}$ wurden gehoben $120 \mathrm{gr}$,

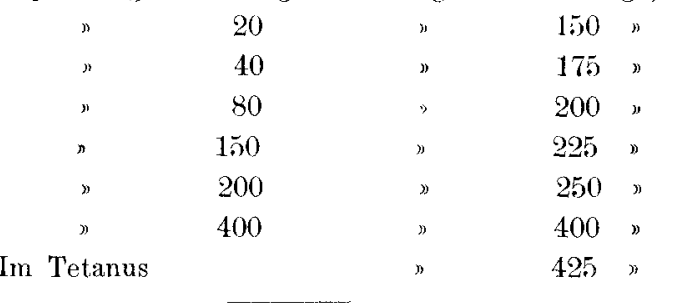

Rana temporaria; mittelgrosses Männchen, durch Aether getöllet.

Bei Spannung mit $10 \mathrm{gr}$ wurden gehoben $125 \mathrm{gr}$,

$\begin{array}{lllll}" & 20 & n & 190 " \\ " & 40 & n & 230 & " \\ " & 80 & n & 260\end{array}$


Bei Spannung mit $150 \mathrm{gr}$ wurden gehoben $310 \mathrm{gr}$,

$\begin{array}{crrrr}\rtimes & 200 & » & 350 & \\ \text { Im Tetanus } & 950 & » & 950 & \\ & & & 1000 & \end{array}$

Eine offenbar hierher gehörige Thatsache erwähnt schon Valentin (Lehrbuch der Physiologie Bd. 2). Er machte nämlich die Beobachtung, dass der Gastrocnemius des Frosches nur dann das Maximum seiner absoluten Kraft entfalte, wenn der Ansatz der Achillessehne das Unterschenkeltarsalgelenk um einige Millimeter iiberragt, wodurch er den Muskel uber seine gewöhnliche Länge dehnte. Diese Differenz in der Grösse der absoluten Kraft eines Muskels erklärte er aus dem Kraftverlust, den ein sich selbst überlassener Muskel durch die Contraction seiner Fasern erleide. In Wirklichkeit ist diese Differenz aber bedingt durch die stärkere Spannung des Muskels, welche mit der Grösse der absoluten Kraft beinahe in geradem Verhältniss steht.

Zur weiteren Beleuchtung jener eigenthtimlichen Eigenschaft des Muskels, selbst bei einem einmaligen Reiz eine um so grössere absolute Kraft zu erreichen, je stärker er gespannt wird, erlaube ich mir auf die Versuche 1 und $2 \mathrm{zu}$ verweisen, die sich auf schnelle und langsam arbeitende Muskeln beziehen, und zwar auf ein paar sicherlich gleichartig gebaute Muskeln, nämlich den Gastrocnemius des Frosches und den der Kröte.

Am tibersichtlichsten treten diese Gesetzlichkeiten zu Tage, wenn man das Anwachsen der absoluten Kräfte des Gastrocnemius der Kröte einerseits und der des Frosches andererseits in Form einer Curve zeichnet, wobei die Spannungen des Muskels den Abseissen und seine absoluten Kräfte den Ordinaten entsprechen. Fig. $4 a$ auf S. 364 zeigt das Anwachsen der absoluten Kraft beim Froschmuskel, Fig. $4 b$ dasselbe bei dem viel kleineren Krötenmuskel.

Aus der Vergleichung vorstehender beider Curven ersieht man, dass der schnell arbeitende Froschmuskel in Folge geringer Spannung viel schneller auf der höchsten Höhe seiner absoluten Kraft ankommt, als der langsam arbeitende Krötenmuskel; dieser passt sich viel besser mit seiner Spannung der gewissermaassen in ihn hinein gelegten Spannung an und ibertrifft dieselbe immer nur um ein Weniges. (Siehe die Zahlen von Versuch 9, nach welchem Figur $4 a$, und von Versuch 10, nach welchem Figur $4 b$ gezeichnet ist.) Der schnelle Froschmuskel dagegen erreicht binnen 
kürzester Zeit, wenn er nur ein wenig gespannt wird, sehr bald seine maximale, absolute Kraft, arbeitet also bei weitem nicht so

Fig. $4 \%$. Fig. 4.6 .

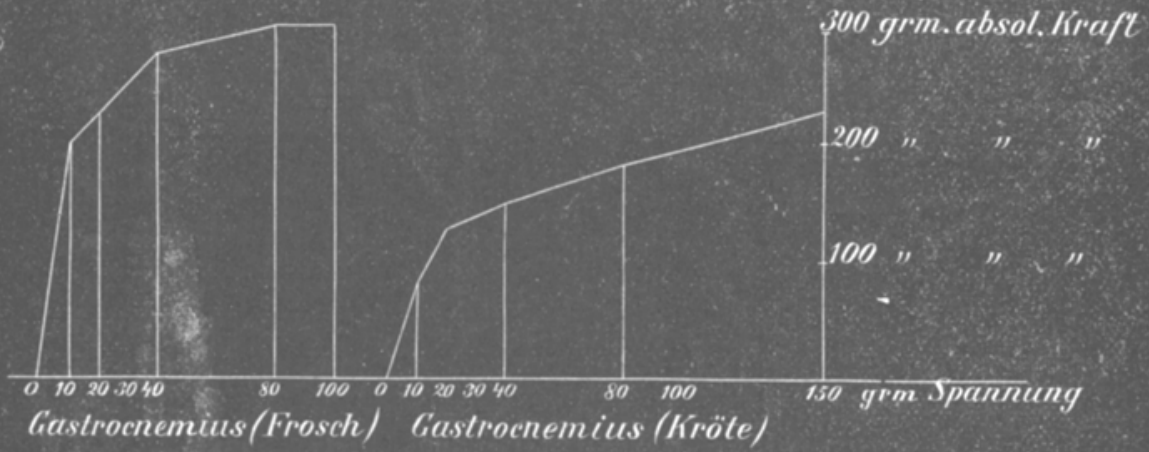

hanshailterisch wie der Krotenmusel Gamy ahuliehe Thatsachen treten uns nun andi entgegen an den bangran wnd selnoll arbeitenden Muskeh ein und desselben Thieres, Fin ausserordentich schneller Maskel ist z. B. der Seminembranosus mud denentsprechend verhält sich auch das Anwachsen seiner absoluten Kräte mit steigender spannung (vergleiche hierzu die /ahlen von Versuch 4 and Fig. 5). Fine älnliche Curve wie der semimembranosus gibt auch der Quadriceps. Ein Gegenstäck hierzu bildet der tragge Rectus abdominis. Vergleiche hierza Fig. 6, welehe nach Verach s gezeichnet ist.

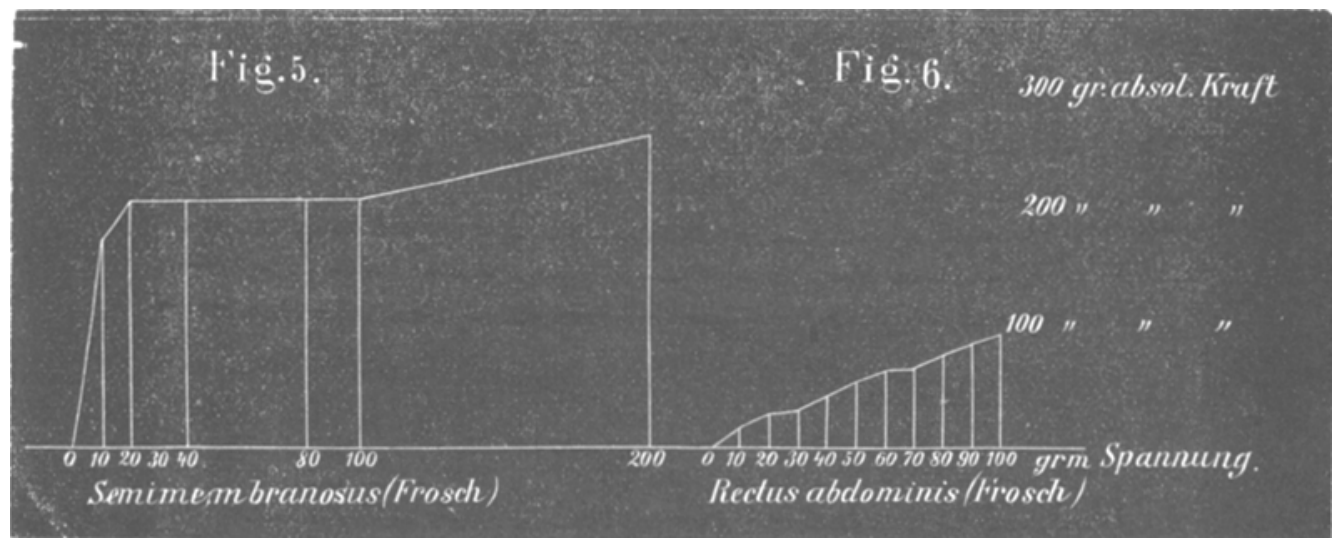


Wenn oben behauptet ist, dass die absolute Kraft eines Froschmuskels bei Znekung hinter der im Tetanus kaum zurickbleibe, so gilt das wesentlich nur von den schnell arbeitenden Muskeln des Frosches, dagegen nicht z. B. vom Gastrocnemius der Kröte; dieser vielmehr leistet im Tetanus bedeutend mehr als in der Zuckung. Um störende Ermüdungserscheinungen auszuschliessen, wurde der Muskel sofort zur maximalen Zuckung bezw. zum maximalen Tetanus gebracht. Hier einige Beispiele.

12. Versueh.

19. Juli 1887. Rana escul. Gastrocn. Länge $28 \mathrm{~mm}$, Gewicht $0,9 \mathrm{gr}$. Bei sofort eingeleitetem maximalen Tetanus wurden $700 \mathrm{gr}$ Gewicht gehohen und bei maximaler Zuckung ebenfalls $700 \mathrm{gr}$. Bei Reduktion auf 1 qen resultirt daher für Zuckung und Tetanus ein Werth von 2306,4 gr.

13. Versuch.

Kröte. Gastrocn. Länge $20 \mathrm{~mm}$; Gewicht 1,2 gr. Bei sofortigem maximalen Tetanus wurden $1200 \mathrm{gr}$ abgehoben, also absolute Kraft pro $1 \mathrm{gcm} 2116,4 \mathrm{gr}$ im Tetanus; bei sofortiger maximaler Zuckung wurden $800 \mathrm{gr}$ abgehoben, also absolute Kraft pro 1 qem 1410,9 in Zuckung.

14. Versuch.

20. Juli 1887. Rana tempor. Gastrocn. Länge $30 \mathrm{~mm}$; Gew. 0,75 gr. Bei sofortigem maximalen. Tetanus wurden $1000 \mathrm{gr}$ abgehoben und bei sofortiger maximaler Zuckung ebenfalls $1000 \mathrm{gr}$, also pro $1 \mathrm{qcm} 4237,2 \mathrm{gr}$ in Zuckung und Tetanus.

15. Versuch.

Kröte. Gastroen. Länge $22 \mathrm{~mm}$; Gowicht $0,4 \mathrm{gr}$. Bei sofortigem maximalen Tetanus wurden $900 \mathrm{gr}$ abgehoben, also pro 1 qcm 5263,1 $\mathrm{gr}$ im Tetanus, bei sofortiger maximaler Zuckung wurden $600 \mathrm{gr}$ abgehoben, also pro 1 q $\mathrm{cm} 3508,7 \mathrm{gr}$ in Zuckung.

Sehliesslich möchte ich noch mit ein paar Worten darauf hinweisen, dass ich mit dem Griitzner'sehen Myographium (s. dieses Archiv Bd. 41, S. 281) auch die zeitliche Entwickelung der absoluten Kraft unter versehiedenen Anfangsspannungen beobachtet habe, indem ich mit genanntem Apparat ähnlich wie es Fick gethan, isometrische Curven, aber nicht von der Anfangsspannung des Mnskels $=$ Null, sondern von einer bestimmten positiven Anfangsspannung aus zeichnete. Dic hierbei gewonnenen Ergebnisse sind höchst beachtenswerth, indem auch sie zcigen, dass der Muskel 
eine viel bedeutendere und länger dauernde Spannung erreicht, also den Myographionhebel viel höher und länger hebt, je mehr er von Haus aus gespannt ist, dass er aber andererseits eine verhältnissmässig sehr geringe und eine nur kurze Zeit dauernde Spannung aufweist, wenn er von Haus aus wenig oder gar nicht gespannt ist. Steigt man mit der Anfangsspannung über eine gewisse Grenze hinaus, so wird die isometrische Curve zweigipfelig, die Entwicklung der absoluten Kraft erfolgt also absatzweise, nicht continuirlich. Wird schliesslich die Anfangsspannung gar zu gross, so iberwindet sie der Muskel bei seiner Reizung nicht mehr. Die Curve verläuft horizontal. Die genaueren Einzelheiten gedenkt Herr Prof. Grützner nächstens ausführlicher mitzutheilen.

Sehr gern hätte ich nun auch noch die absolute Kraft der Warmbliitermuskeln und ihre Abhängigkeit von ihrer Anfangsspannung u. s. w. untersucht. Leider aber sind die technischen Schwierigkeiten hier viel bedeutender als bei den isolirten und auf bequeme Weise befestigten Froseb- oder Krötenmuskeln. Meine Zeit reichte leider nicht weiter, so dass ich ïber die ersten tastenden Versuche nicht hinausgekommen bin. Insofern aber ist schon eine Uebereinstimmung mit den Thatsachen an den verschiedenen Kaltbliutermuskeln zu beobachten, als, wie die Versuche von Grützner ${ }^{1}$ ) gelehrt, der langsame rothe Süngethiermuskel im Tetanus ausserordentlich viel mehr leistet, als der weisse flinke Säugethiermuskel ${ }^{2}$ ). Entsprechend seinen Leistungen (Heben von Lasten) wird sich aller Wahrscheinlichkeit nach auch seine absolute Kraft gestalten, worüber weitere Untersuchungen bald Aufschluss geben diurften.

Wie allgemein die Verbreitung flinker und langsamer Muskelfasern, die aller Wabrscheinlichkeit nach auch physiologisch ungemein gleichartig sind, im Thierreich ist, das ersieht man unter Anderem aus den schönen Untersuchungen von Rollett ${ }^{5}$ ) u. a.,

1) Breslauer ärztliche Zeitschrift; 9. Jahrgang 1887, Nr. 1.

2) Dem Tetanus entsprechend verhält sich, wie neuere interessante Untersuchungen von Max Bierfreund (dieses Archiv Bd. XLIII, S. 195) ergeben haben, auch der rothe Muskel in der Todtenstarre. Die Starre tritt bei den rothen Muskeln viel später ein und erreicht einen viel bedeutenderen Grad, als hei den weissen.

3) Denkschriften der mathematisch-naturwissenschaftlichen Classe der Wiener Academie, Bd. 49 1885, Bd. 51 1885, Bd. 531887. 
der diese verschiedenen Muskeln an den Insekten beobachtet und auf das Sorgfältigste und Eleganteste in ihren physiologischen Eigenschaften studirt hat. Auch $\mathrm{F}_{0}{ }^{1}{ }^{1}$ ) findet bei Mollusken zweierlei verschiedene (glatte) Muskeln, 1) solche, deren Zellen nur aus parallelen Fibrillen bestehen und 2) solche, deren Fibrillen spiralig gewunden sind und vielen Untersuchern den Eindruck von Querstreifen gemacht haben. Wahrscheinlich sind erstere langsame, letztere flinke Muskeln.

Zum Scbluss sei es mir gestattet, Herrn Prof. Grützner für die Anregung zu dieser Arbeit und die gütige Unterstiutzung, die er mir bei der Ausfïhrung derselben zu Theil werden liess, meinen innigsten Dank auszusprechen.

(Organ. Laboratorium der techn. Hochschule und Chem. Laborat. des patholog. Institutes zu Berlin.

\section{Ueber Anthrarobin und Chrysarobin.} Von

Dr. Theodor Weyl in Berlin.

Das Chrysarobin hat in dem Anthrarobin, welehes Herr G. Behrend ${ }^{2}$ ) auf Empfehlung des Herrn C. Liebermann ${ }^{3}$ ) dem A rzneimittelschatze einverleibte, einen - wie es scheint - fast ebenbiirtigen Mitstreiter gefunden.

Ich schildre im Folgenden Versuche ${ }^{4}$ ), die sich mit den Wirkungen und Schicksalen der genannten beiden Stoffe bei cutaner und interner (subcutaner und stomachaler) Application be-

1) Comptes rendus, T. 106, p. 306, 1888.

2) G. Behrend: Vierteljahrsschr. f. Dermat. u. Syphil. 1888, 201/208 und Therapeut. Monatshefte 1888, 101/103.

3) C. Liebermann: Ber. d. dentsch. chem. Ges. 1888, 447/452 und Therapeut. Monatshefte 1888, 145/148.

4) Die Anthrarobin-Versuche habe ich auf Wunsch des Herrn Prof. Liebermann angestellt. Demselben Herrn verdanke ich auch eine Probe sehr reinen Chrysarobins. Das Anthrarobin stellten die Herren Dr. Jaffé und Darmstaedter freundlichst zur Verfügung. Das Chrysarobin war von Gehe \& Co. bezogen. 